\title{
Sarcomas and Hypertrophic Pulmonary Osteoarthropathy
}

\section{Samuel J. Harris*, Lucy Dumas and Charlotte Benson}

Sarcoma Unit, Department of Medical Oncology, Royal Marsden Hospital, London, UK

\section{Introduction}

Hypertrophic pulmonary osteoarthropathy (HPOA) is a syndrome defined by clubbing of the fingers and toes, arthritis and periostitis [1]. It was first described by Bamberger in 1889 and Pierre Maire in 1890 although clubbing, was recognized by Hippocrates 2500 years ago [2,3]. It is also known as Marie-Bambeger Disease. It has long been associated with Pulmonary Malignancies such as Non-Small-CellLung Cancer and mesothelioma as well as supporative lung disease and cyanotic congenital heart disease amongst other causes [4]. It is not commonly associated with sarcomas, either primary or metastatic. Here we present two cases of thoracic sarcomas that presented with HPOA and or clubbing. We provide a review of the literature with regard to Sarcoma and HPOA as well as a discussion of the underlying pathophysiology.

\section{Case 1}

A 30 year old gentleman who presented with a six week history of fatigue and arthralgia. Joint pains had begun in his knees but progressed to involve the wrists, ankles and fingers with marked joint swelling. $\mathrm{He}$ also reported a several week history of worsening left sided chest pain. On examination he was noted to have marked clubbing. A plain chest film demonstrated two lung masses, one on the left and the second on the right. Plain films of both ankles demonstrated bilateral periosteal reactions around the distal and fibulae consistent with hypertrophic pulmonary osteoarthropathy (Figure 1). A subsequent CT chest revealed pleurally based masses throughout the left hemithorax measuring up to $7.9 \mathrm{~cm}$ and radiological representative of an entirely separate etiology having a solid, vascular, infiltrative appearance. There was an associated left pleural effusion and resulting collapse of the left lower lobe. There was no abnormality below the diaphragm and no other bony abnormalities seen on CT. Biopsy of the lesion revealed changes consistent with a translocation negative Ewing's sarcoma, or Periperheral Neuroectodermal Tumour

A PET-CT (Figure 2) confirmed no other sites of disease and he was therefore commenced on induction chemotherapy as per standard therapy for a chest wall Ewing Sarcoma comprising of Vincristine, Doxorubicin, Cyclophosphamide alternating with Ifosfamide and Etoposide (VDC/IE) for four cycles with a view to surgical resection. He tolerated chemotherapy well and had a rapid resolution of arthralgia, chest pain and his clubbing which continued to improve though treatment. He went onto complete four cycles of VDC/IE with subsequent chest wall resection and lingulectomy demonstrating no viable tumour. By the time he commenced his adjuvant chemotherapy, his clubbing and arthralgia had fully resolved however the florid periosteal reaction affecting the distal tibia and fibula remains unchanged on most recent plain films despite clear and definite clinical resolution.

\section{Case 2}

A 69 year old lady was referred to our unit with an incidental finding of a mass in the right lower lobe associated with a large right sided effusion. This had come to light incidentally, following an admission to her local hospital with severe diarrhoea. Her admission CXR was clearly abnormal and this led to a CT CAP which demonstrated a $13 \mathrm{~cm}$ mass in the right lower lobe pushing down onto the diaphragm with some smaller satellite nodules surrounding the mass but no other distant metastatic disease. She also was also noted to have marked clubbing of the fingers which she reports has developed more recently. She had a past medical history of smoking and known cardiac disease but no pre-existant respiratory conditions and prior to her admission had been fit and well with no limitations on her exercise tolerance. Biopsies taken from the mass revealed dedifferentiated liposarcoma, with the characteristic Murine Double Minute 2 (MDM2) amplification. There was considerable time between first presentation and the review at our institution and interval imaging over this 4 month period of time revealed little change in the mass. Given the lack of symptoms and seemingly indolent disease, the patient opted for a watch and wait approach to treatment (Figure 3)

\section{Sarcoma and HPOA/Clubbing}

HPOA and or clubbing is infrequently seen in patients with sarcomas. However, previous single cases reports have been reported of HPOA with multiple different sarcoma subtypes including,

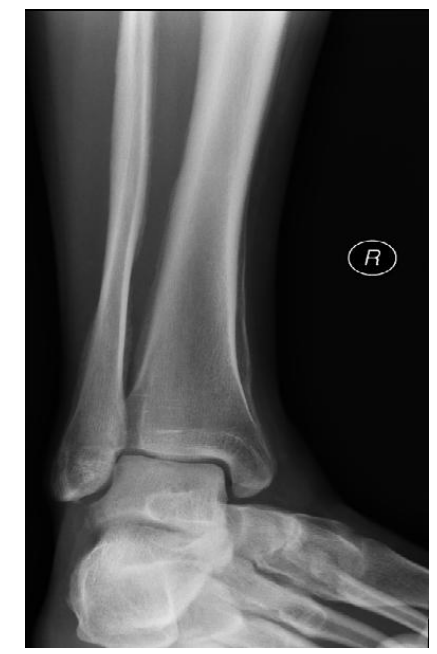

Figure 1: Plain X-ray of ankle and tibia, showing characteristic periosteal changes of HPOA.

*Corresponding author: Samuel J. Harris, Sarcoma Unit, Department of Medical Oncology, Royal Marsden Hospital, Fulham Road, London, SW3 6JJ UK, Tel: +44 7472297878; E-mail: Samuel.Harris@rmh.nhs.uk

Received August 21, 2014; Accepted September 28, 2014; Published September 30, 2014

Citation: Harris SJ, Dumas L, Benson C (2014) Sarcomas and Hypertrophic Pulmonary Osteoarthropathy. J Carcinog Mutagen 5: 193. doi:10.4172/21572518.1000193

Copyright: @ 2014 Harris SJ, et al. This is an open-access article distributed under the terms of the Creative Commons Attribution License, which permits unrestricted use, distribution, and reproduction in any medium, provided the original author and source are credited. 


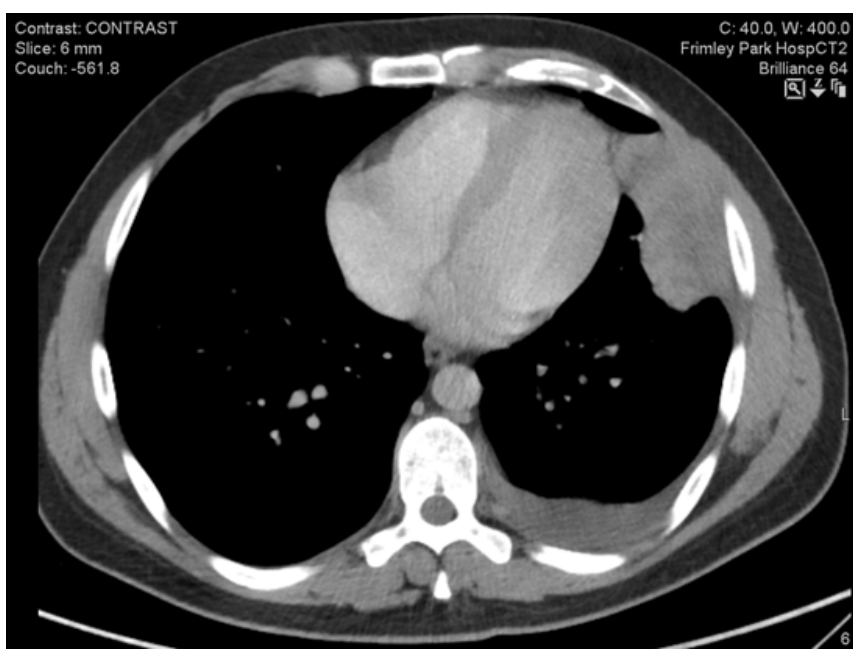

Figure 2: Axial CT showing Left chest wall Ewing's Sarcoma.

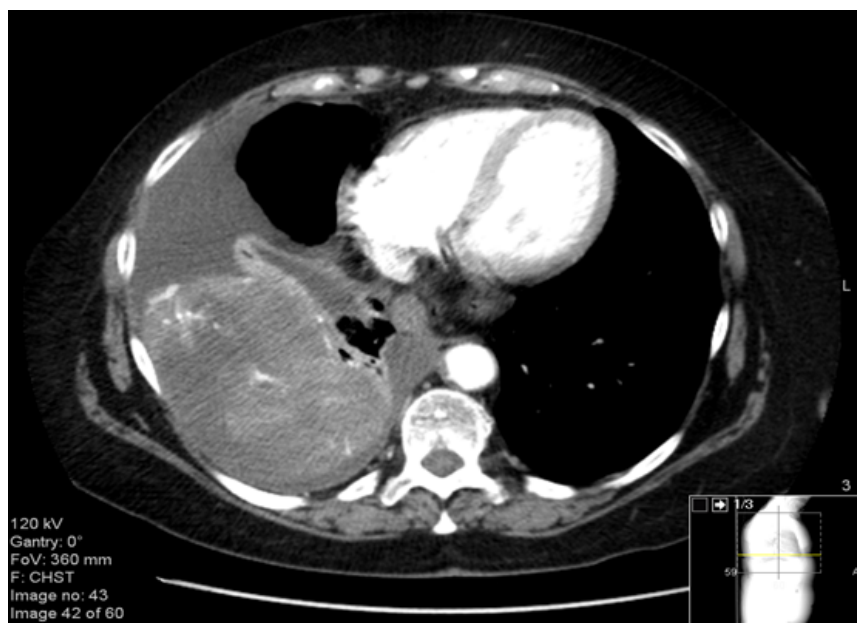

Figure 3: Axial CT image of Right Lower Lobe De-Differentiated Liposarcoma.

but not limited to fibrosarcoma, leiomyosarcoma, osteoarcomas, rhabdomyosarcoma and phyllodes cancer [5-9]. The association remains rare with only a small number of cases having been reported. Only one other case of Ewings sarcoma of the chest wall has previously been reported [10]. To our knowledge liposarcoma has not been previously described with clubbing or HPOA. Nearly all of the cases reported involved either primary pulmonary lesions or secondary pulmonary deposits. Many of the cases reported show a resolution in symptoms with successful treatment of the underlying malignancy with some reporting recurrence of symptoms with recurrence of the sarcoma.

Although the association of sarcoma and HPOA is not frequent, Solitary Fibrous Tumour [SFT] is one particular subtype of sarcoma that has been more commonly associated with HPOA. SFT is a vascular malignancy that commonly occurs in the pleura and has reported to cause clubbing in approximately $15 \%$ of pleural cases [11].

Sarcomas metastatic to lung may be more likely to cause clubbing than other malignancies with pulmonary metastases. A case series of 34 patients with pulmonary metastases associated with HPOA found 25 out these to be from secondary sarcomas [12]. Other series have shown similar result with up to half of cases from pulmonary metastases relating to underlying sarcoma primary. Despite this the overall incidence remains rare [13].

\section{Pathophysiology of Sarcoma}

HPOA and clubbing are thought to be related in that they are different endpoints of the same underlying pathophysiologic process. Numerous theories exist as to the mechanism; however our knowledge on this process remains incomplete. Pulmonary shunting, chronic hypoxia, altered vagal tone, platelet clumping and paraneoplastic growth factors such as Vascular Endothelial Growth Factor (VEGF) or Prostaglandin E have all been implicated [14].

The presence of a pulmonary Right to Left shunt seems to be an important factor in the development of HPOA/Clubbing. Congenital Cyanotic Heart disease has long been associated with the phenomena and patients that have their shunt reversed often have improvement in symptoms. Likewise, it has been reported that patients with patent ductus arterious, who have blood entering the right descending aorta from the pulmonary artery, develop clubbing in the feet but not hands [15]. Patients with pulmonary or thoracic masses are thought to develop shunting because of new and aberrant vessel formation. Perhaps highlighting why, sarcomas, which are often vascular tumours have a higher tendency to develop the condition as metastatic disease.

An important theory behind the cause of HPOA is the release of angiogenic and other growth factors in the distal limbs. This is thought be secondary to megakaryocytes avoiding fragmentation into platelets in the pulmonary circulation because of the intrapulonaruy shunting. The megakaryocytes then reach distal sites where they are trapped and release growth factors such as Vascualar Endothelial Growth Factor (VEGF), Platelet Derived Growth factor, transforming growth factor (PDGF). These peptides are all angiogenic, stimulating capillary growth, and increased blood supply. PDGF and VEGF have also been implicated in stimulating osteoblastic and fibroblastic activity. The combination of new blood vessel formation, activation of the endotheium, new bone formation and fibroblastic activity then results in the classic findings of nail bed changes, interstitial oedema and swelling of clubbing and the periostitis of HPOA $[14,15]$.

Other factors seem to play a role as well. Chronic hypoxia is known to stimulate VEGF activity and macrophage activity, although hypoxia on its own does not seem to cause HPOA. Vagal tone may also be important, is highlighted by the success in vagotomy in reversing HPOA. Prostaglandin E2 may also play a role, as pE2 levels have been shown to higher in patients with clubbing. It is thought that the pulmonary shunting may prevent the normal inactivation of $\mathrm{pE} 2$ and other circulating factors in the pulmonary circulation [15].

Vascular Endothelial Growth Factor has also been implicated in the pathogenesis of HPOA through direct tumour production. Cases have been reported showing elevated circulating levels of VEGF in patients with clubbing associated with lung cancer. In these cases, following treatment of the lung cancer, alongside improvement in the clubbing a fall in the circulating VEGF was also noted. Certainly, many VEGF associated tumours such as mesothelioma have been linked with HPOA and clubbing. However, extrapulmonary tumours associated with VEGF do not seem to cause clubbing/HPOA suggesting that other factors alongside VEGF must also be important [15].

\section{Conclusion}

The two cases we have discussed highlight the rare, but recognized 
Citation: Harris SJ, Dumas L, Benson C (2014) Sarcomas and Hypertrophic Pulmonary Osteoarthropathy. J Carcinog Mutagen 5: 193. doi:10.4172/2157-2518.1000193

association between sarcomas and HPOA. Sarcoma, hence should not be discounted as a diagnosis on the basis of presence of the signs or symptoms of HPOA. We are reminded of the importance of the clinical finding of HPOA/clubbing in prompting further investigation for underlying malignancy.

\section{Reference}

1. Yeung SC, Habra MA, Thosani SN (2011) Lung cancer-induced paraneoplastic syndromes. Curr Opin Pulm Med 17: 260-268.

2. Bamberger E (1889) Sitzung der KK Ges der Aerzte in Wien. Wien Klin Wschr 2: 226.

3. Hippocrates, Edward Theodore Withington, Paul Potter (1849) Hippocrates: Prognostic: Regimen in Acute Diseases, Sacred Disease, Art, Breaths, Law, Decorum, Physician, Dentition (Loeb Classical Library), Harvard University Press, Cambridge, USA

4. Qian X, Qin J (2014) Hypertrophic pulmonary osteoarthropathy with primary lung cancer. Oncol Lett 7: 2079-2082.

5. Andres R, Saenz A, Mayordomo J I, Zaballos P, Isla D, et al. (2003) Case 4. Hypertrophic osteoarthropathy associated with pulmonary metastasis of uterine leiomyosarcoma. Journal of clinical oncology 21: 3540-3541.

6. Garganese MC, De Sio L, Serra A, De loris MA, Bock C, et al. (2009) Rhabdomyosarcoma associated hypertrophic osteoarthropathy in a child: detection by bone scintigraphy. Clinical nuclear medicine 34: 155-157.
7. Flueckiger F, Fotter R, Hausegger K, Urban C (1989) Hypertrophic osteoarthropathy caused by lung metastasis of an osteosarcoma. Pediatr Radiol 20: 128-130.

8. Collinson FJ, Bilous AM, Kefford RF (2004) Hypertrophic osteoarthropathy from pulmonary metastatic phyllodes tumour of the breast. Med J Aust 181: 279.

9. Banerjee AK, Carvalho P (1991) Hypertrophic pulmonary osteoarthropathy due to a pulmonary metastasis from a soft tissue sarcoma. Clin Nucl Med 16: 270272.

10. Murphy C, Sharial SNMM, Brennan N, Lee G, Moylan E, et al. (2008) Hypertrophic osteoarthropathy leading to the diagnosis of primitive neuroectodermal tumour (PNET). Acta oncologica 47: 983-986

11. Rena O, Filosso PL, Papalia E, Molinatti M, Di Marzio P, et al. (2001) Solitary fibrous tumour of the pleura: surgical treatment. Eur J Cardiothorac Surg 19: 185-189.

12. AUFSES AH, AUFSES BH (1960) Hypertrophic osteoarthropathy in association with pulmonary metastases from extrathoracic malignancies. Dis Chest 38 399-402.

13. Yacoub MH, Simon G, Ohnsorge J (1967) Hypertrophic pulmonary osteoarthropathy in association with pulmonary metastases from extrathoracic tumours. Thorax 22: 226-231.

14. Dickinson CJ (1993) The aetiology of clubbing and hypertrophic osteoarthropathy. Eur J Clin Invest 23: 330-338.

15. Rutherford JD (2013) Digital clubbing. Circulation 127: 1997-1999. 